Some authors are pure, academic scholars, others have been very closely involved in the negotiations of the Convention and are now participating in the implementation process; some are both. For example Vincente Sánchez, one of the editors, was the Chairman of the Intergovernmental Negotiating Committee for the Convention and is now the President of the Bureau of the Intergovernmental Committee preparing the first Conference of the Parties. Veit Koester, co-author of the chapter on access to genetic resources, chaired Working Group II during the negotiation process, which dealt inter alia with access to biodiversity and related technologies. Ashish Kothari from the Indian Institute of Public Administration is following the international process on biological diversity from a scientific as well as from a political point of view, presenting the so called "third world perspective" as an NGO-representative at the negotiations.

For anyone who wants to become familiar with the political and legal problems at stake in implementing the Convention on Biological Diversity, this book is a valuable, even indispensable contribution. It is one of the few volumes addressing the abundant problems of the Convention, reflects the wide-ranging debate in the search for solutions to these problems and provides proposals for their solution.

Gudrun Henne

\title{
Erik Franckx
}

\section{Maritime Claims in the Arctic - Canadian and Russian Perspectives}

Martinus Nijhoff Publishers, Dordrecht, 1993, 330 S., $£ 95.00$

Wenn die internationale Presse über Umweltrisiken in der Arktis berichtet, dann geht es in der Regel um Ölunfälle im hohen Norden Rußlands, um versenkten Nuklearschrott und andere Schreckensmeldungen. Die geplante Einrichtung von arktischen Schiffahrtswegen auf der Nord-Ostpassage oder der Nord-Westpassage ist nur wenigen Beobachtern bekannt.

Erik Franckx hat mit dieser überarbeiteten Ph.D.-Dissertation ein "vernachlässigtes" regionales Meer seerechtlich dargestellt und damit einen wertvollen Beitrag zur Aktualisierung des Seerechts zum Zeitpunkt des Inkrafttretens des neuen UN-Seerechtsübereinkommens geleistet.

Das Buch ist nicht nur - wie der Titel vermuten läßt - eine rechtsvergleichende Darstellung der kanadischen und russischen Gebietsansprüche auf die Wasser- und Eisflächen der Arktis, sondern eine schiffahrtsrechtliche Analyse der beiden großen arktischen Seewege in den Pazifischen Ozean. Die Rechtsfragen anderer Nutzungskonflikte (Ölgewinnung, Meeresforschung, militärische Fragen) werden leider nur knapp behandelt, aber in dem ungewöhnlich umfangreichen Fußnotenapparat (ein Drittel des Buches nehmen die Fußnoten ein) ausreichend berücksichtigt. 
Die im Mittelpunkt stehenden Verkehrsrechte veranlaßten Kanada und die Sowjetunion zu einer stark interessengeprägten und häufig völkerrechtswidrigen Praxis, die Franckx kritisch, aber fair mit all ihren Widersprüchen und internationalen Verwicklungen analysiert. Während Kanada vorzugsweise die Rechtsinstitute der Umweltzone, der Basislinien und der Meerengen strapazierte, pflegte die Sowjetunion insbesondere die Sektorentheorie, das Konzept der "historischen Meere" und eine systematische Überdehnung des Seerechts in der nationalen Gesetzgebung. Den USA gebührt das Verdienst, mit diplomatischer Kunst und gelegentlichen Fahrten ihrer Schiffe freie Durchfahrtsrechte praktiziert zu haben, auch wenn Franckx wenig Sympathie für diese Art Ausdauer zeigt.

Die negative Haltung Kanadas und Rußlands zur Öffnung der Arktis weicht in den letzten Jahren, wie Franckx am Schluß seiner Studie zeigt, der Erkenntnis, daß eine umweltverträgliche polare Schiffahrt Vorteile bieten kann, und daß die Erforschung und der Schutz der Arktis ein großes Thema der Zusammenarbeit wird. Dazu bedarf es der Kenntnis der Rechtslage in der Arktis und der bi- und multilateralen Instrumente der regionalen Zusammenarbeit der interessierten Staaten, die der Verfasser sehr sorgfältig, mit großem Engagement und eigenen Empfehlungen aufgearbeitet hat.

Uwe Jenisch

\section{Norman Paech / Gerhard Stuby \\ Machtpolitik und Völkerrecht in den internationalen Beziehungen}

Nomos Verlagsgesellschaft, Baden-Baden, 1994, 878 S., DM 148,--

Mit ihrem dickleibigen Werk beabsichtigen die beiden in Hamburg bzw. Bremen als Juristen lehrenden Autoren, wie sie im Vorwort sagen, "zumindest einen Zugang zum Verständnis für die Wirkungsweise von Völkerrecht in den internationalen Beziehungen zu eröffnen" (3). Sie nähern sich mithin von der rechtswissenschaftlichen Seite aus jenem Grenzland zwischen Völkerrechtslehre und politikwissenschaftlicher Lehre von den internationalen Beziehungen, das von letzterer Seite aus, oder, wie die Autoren noch allgemeiner, aber zu Recht feststellen, von sozialwissenschaftlicher Seite aus eher selten beschritten wird. Ein solches Buch, mit dem die Autoren nicht "den vielen vorhandenen Völkerrechtslehrbüchern ein weiteres hinzuzufügen" versuchen, sondern "das Entstehen der Völkerrechtsnormen aus dem historischen Entstehungsprozeß des heutigen internationalen Systems heraus verständlich zu machen und in seiner aktuellen Funktion im gegenwärtigen internationalen System darzustellen" (5) beabsichtigen, ist hoch willkommen. Obwohl dieser Anspruch, ungeachtet des Umfangs, im Ergebnis nicht zur vollen Zufriedenheit des Rezensenten eingelöst wurde, ist gleichwohl ein Werk von hohem Nutzen entstanden, allerdings wohl mehr für in völkerrechtlichen Dingen weniger vorgebildete politikwissenschaftliche LeserInnen, weniger für JuristInnen. Der Grund hierfür liegt darin, daß das Buch, das kein Völkerrechtslehrbuch sein will, sich über weite Strecken unvermeidbarer- 